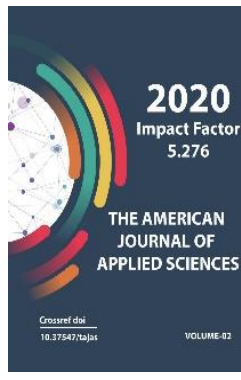

Copyright: Original content from this work may be used under the terms of the creative commons attributes 4.0 licence.

\section{Methods For Generation Of Cluster Particles Of Different Stochiometry}

\author{
Khozhiev Sh.T. \\ Academician Sadykov Institute Of Bioorganic Chemistry, Uzbekistan \\ Kosimov I.O. \\ Academician Sadykov Institute Of Bioorganic Chemistry, Uzbekistan \\ Murodkobilov D.M. \\ Tashkent State Technical University, Uzbekistan \\ Gaibnazarov B.B. \\ Tashkent State Technical University, Uzbekistan \\ Bekmirzaev.J.N. \\ Academician Sadykov Institute Of Bioorganic Chemistry, Uzbekistan \\ Mutalliev.L. Z \\ Academician Sadykov Institute Of Bioorganic Chemistry, Uzbekistan
}

\title{
ABSTRACT
}

The main purpose from this paper, in semiconductor technology silicon oxide is to study notable of the surface and catalysis process. As a result, cluster fragmentation processes, we obtained information about the emission of the clusters and the chemical and physical properties of the clusters. We found during the study that oxide clusters are heterogeneous clusters. In heterogeneous clusters, the bonds in the main chain are different from the bonds in the side chain, as in organic polymers. We tried to prove on the basis of the analysis of experimental data that the formation, excitation and molecular decomposition of $\mathrm{Sin}_{n} \mathrm{O}_{2 n+1} \mathrm{H}_{\mathrm{k}}-(\mathrm{k}=1-3)$ cluster ions are based on the model of the combinatorial synthesis mechanism.

\section{KEYWORDS}

Vibrationally,Nanoelectronics,Microanalyzer,Hydrogenated.

\section{INTRODUCTION}

The sputtering of targets by beams of accelerated primary ions is one of the most productive methods for generating cluster particles is the sputtering of targets by beams of accelerated primary ions. It is known that some of the sputtered particles correspond to cluster ions. Clusters are formed in vibrationally excited states and decay on their way from the target to the detector [2]. The discovery of fragmentation of sputtered clusters (Standing's work) has significantly complicated the understanding of the cluster emission mechanism, since their decay affects the measured mass spectra, kinetic energy spectra, and the distribution of internal energies of clusters. Investigation of the processes of cluster decay makes it possible to obtain information both on the emission of clusters and on the chemical and physical properties of these clusters. Physical and chemical properties of a cluster, varying from the properties of atoms to the properties of a solid and depending on the size and structure of the cluster, can serve as a basis for the synthesis of crystals with unique properties that promising for solving urgent problems of modern nanotechnology [3]. Recently studies, considerable attention is paid to clusters of metal oxides, which play an essential role in modern technology - micro- 
and nanoelectronics, surface chemistry, catalysis processes, as well as the search for methods of their synthesis and study of fundamental properties. The study of silicon oxide, which plays an important role in semiconductor technology, catalysis, and surface chemistry, is especially important. It should also be noted that oxides are heterogeneous clusters, and in a heterogeneous cluster, in the main chain the bonds are different from those in the branches, as in organic polymers. In gases, the time between collisions of molecules under normal conditions of 10-10 $\mathrm{s}$ is much longer than the collision time (10-1210-13 s), therefore the reaction act can be considered as a result of an isolated pair collision. Such collisions can lead to a change in the number of particles with an energy exceeding the activation energy of the reaction $E$, and, accordingly, to a violation of the Maxwell-Boltzmann distribution of particles over the energies of their relative motion and internal degrees of freedom. Equilibrium reactions continue relatively slowly, the MaxwellBoltzmann distribution is practically preserved, and the reaction rate can be calculated within the framework of the theory of an activated complex. It has long been known from thermochemical studies that during the sublimation of solid silicon monoxide or silica, the gas phase contains abundant $\mathrm{SiO}, \mathrm{SiO}_{2}$ and oxygen in atomic or molecular form. The presented fragmentation results agree with the experimental observations of other authors. It is known that the dissociation energy of gaseous $\mathrm{SiO} \rightarrow \mathrm{Si}+\mathrm{O}$ and $\mathrm{SiO}_{2} \rightarrow \mathrm{SiO}+\mathrm{O}$ is quite high. It is also known that in gas-phase systems, the particles forming a cluster ion vibrate about an equilibrium situation, the distances of the size of isolated atoms (molecules) of larger than valence electrons remain localized on the atoms. Apparently, most of the clusters formed in a gaseous medium (vacuum) constitute such a cluster. All of the above suggests that atoms and molecules in a cluster largely retain their individual properties. This premise - the preservation of their individual properties by a group of atoms in a cluster - can be used to identify the groups present in a cluster ion, and we used it in studies of gasphase clusters and molecules.

\section{EXPERIMENTAL}

We used the unique capabilities of an ion microanalyzer with double focusing and reverse geometry of Niro - Johnson type to study the formation and fundamental properties of stable hydrogenated silicon oxide clusters $\left(\mathrm{Me}_{n} \mathrm{O}_{\mathrm{m}} \mathrm{H}_{\mathrm{x}}\right)^{ \pm}$or $\left(\mathrm{MeO}_{2}\right) \cdot \mathrm{nOH}^{ \pm}$Fig.1.[9-10]. The clusters will be synthesized by ion sputtering of the silicon surface with oxygen or xenon ions while simultaneously exposing the surface to the atmosphere of water molecules. The dissociation of $\mathrm{H}_{2} \mathrm{O}$ upon interaction with an active oxidized silicon surface with the formation of atomic hydrogen will make it possible to synthesize silicon oxide clusters and hydrogen atoms in the ion impact zone and generate stable cluster configurations $\left(\mathrm{Me}_{n} \mathrm{O}_{\mathrm{m}} \mathrm{H}_{\mathrm{x}}\right)^{ \pm}$or $\left(\mathrm{MeO}_{2}\right) \mathrm{nOH}$. The only property of sputtered cluster particles - to experience a monomolecular decomposition reaction in the gas phase - will make it possible to refine the specific heat, flight times and lifetimes, and the fragmentation reaction rates for such decays, as well as to obtain on their basis such information about the fundamental properties of $\left(\mathrm{MenO}_{m} \mathrm{H}_{\mathrm{x}}\right)^{ \pm}$or $\left(\mathrm{MeO}_{2}\right)$ clusters $\mathrm{nOH}^{ \pm}$as kinetic energies of fragmentation ions and activation energies of decays depending on the number of particles in the cluster. The work also investigates the physics of the processes of capture by stable ionized silicon oxide clusters of a hydrogen atom from water, as well as studies of possible channels in order to the fragmentation of these hydrogenated clusters with the production of hydrogen, both in the ionic fragment and in the neutral one. Also, a comparison is made between the energies of dissociation of water by classical electrolysis and the energies required for the capture and adsorption of hydrogen from water on oxide clusters. This in turn, will also make it possible to reveal the nature of the formation of sputtered clusters. From a practical point of view, the results obtained can be essential for solving urgent problems of the power industry to obtain cheap and environmentally friendly hydrogen fuel from water. 


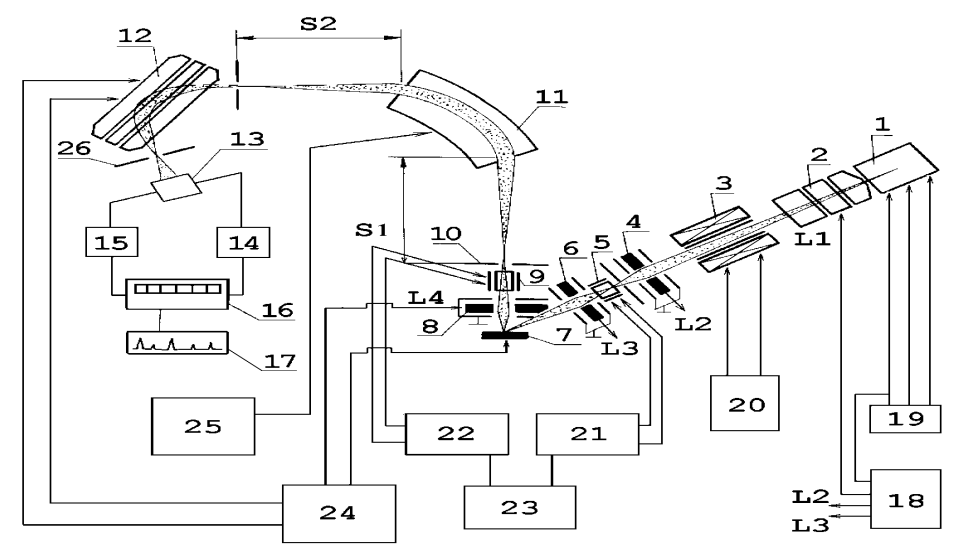

Fig.1

\section{RESULTS AND DISCUSSION}

In the present work, we have identified groups of such clusters that are present in the magic clusters $\mathrm{SinO}_{2 \mathrm{n}+1}^{-}$. It can be seen from the results obtained that fragmentation in the $L_{1}$ zone is similar to the fragmentation reaction in the $L_{2}$ zone, differing only in absolute values. Hence, it follows that the fragmentation reaction for both without field zones has the same nature, and their probability is a function of the lifetime of the corresponding metastable ions - the time from the moment of formation of a metastable ion to its decay.

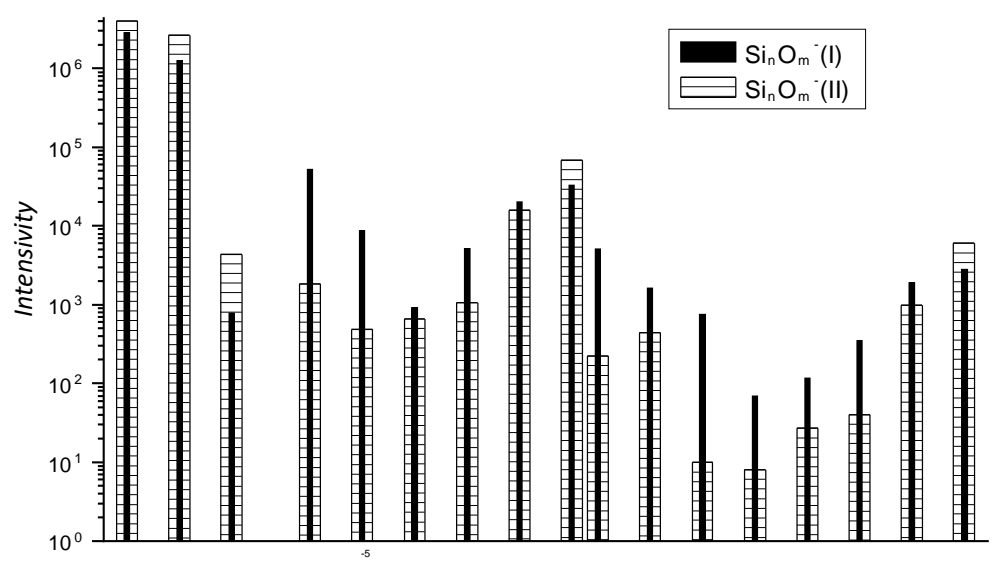

Mass spectra of $\mathrm{Si}_{n} \mathrm{O}_{m} \mathrm{H}_{k^{-}}$clusters ( $n=1-3, m=2 n, 2 n+1, k=0-4$ ) obtained at a current of $\mathrm{O}_{2}{ }^{+}$on the surface of a Si target $\mathrm{I}_{0} \approx 42 \mathrm{nA}(15.07 .2003)$.

$\mathrm{I}$ - residual vacuum $\mathrm{P} \approx 6.5 * 10^{-5} \mathrm{~Pa}$, the surface was cleaned with an ion beam after $\mathrm{H} 2 \mathrm{O}$ was injected into the chamber.

II - inlet into the $\mathrm{H}_{2} \mathrm{O}$ chamber up to $\mathrm{P} \approx 2.5 * 10^{-3} \mathrm{~Pa}$.

Fig.2. 
This makes it possible to assume that similar processes of monomolecular fragmentation of cluster ions take place in the near-surface area of the bombarded target as at distances far from the target.

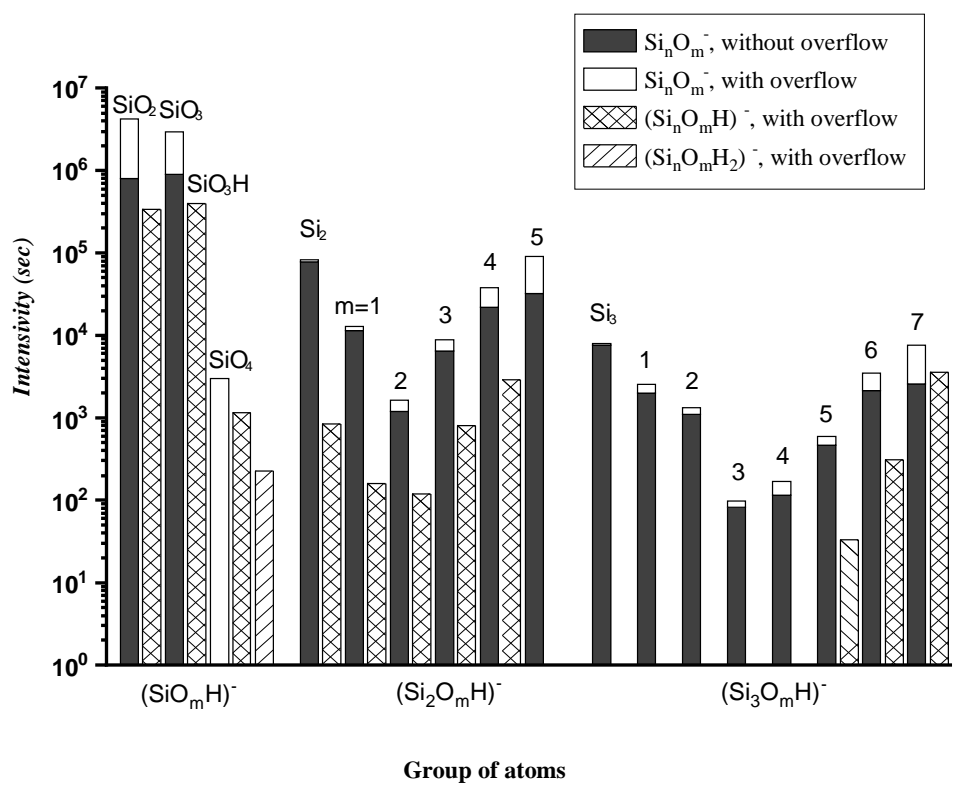

Fig.3.

The study of the mass distributions of $\mathrm{Sin}_{n} \mathrm{O}-2 n+1$ clusters sputtered from the Si surface by $\mathrm{O}_{2}{ }^{+}$ions at different pressures of oxygen and water vapor in the zbombardment chamber, as well as by $\mathrm{Xe}^{+}$ions when oxygen is puffed in, (Fig. 2, 3) displayed that $\mathrm{Sin}_{2 n}$ - and $\mathrm{Sin}_{2 n+1}$ - have an increased intensity in the mass spectra for all methods of their generation, and the absolute values of their intensities when oxygen and water vapor are poured into the chamber at the same pressure differ little. The study of the yields of the $\mathrm{Sin}_{n} \mathrm{O}_{2 n+1} \mathrm{H}_{\mathrm{k}^{-}}$ $(\mathrm{k}=1-3)$ clusters in the case of water vapor admission into the chamber made it possible to draw a conclusion about the decomposition of $\mathrm{H}_{2} \mathrm{O}$ molecules into hydrogen and a hydroxyl group; in this case, hydrogen atoms saturate the existing free bonds in the cluster and stabilize the forming cluster structures, leading to the formation of hydrogenated clusters of the type $\mathrm{Sin}_{2 n+1} \mathrm{H}_{\mathrm{k}^{-}}$.

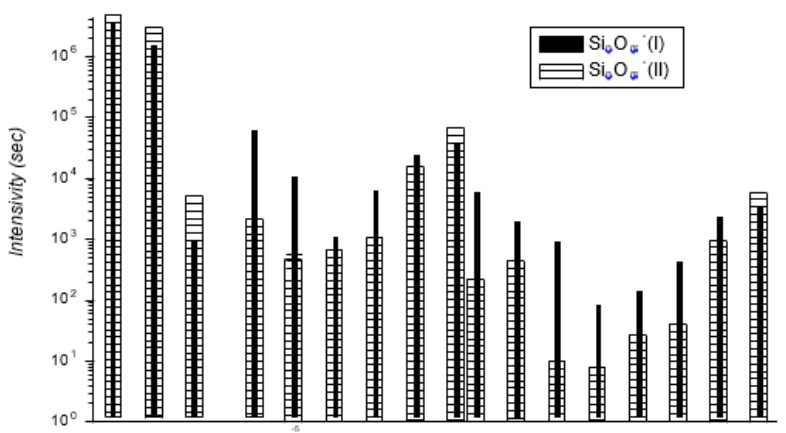

Mass spectra of $\mathrm{Sin}_{n} \mathrm{O}_{m^{-}}(\mathrm{n}=1.2)$ clusters obtained at a current of $\mathrm{O}_{2}{ }^{+}$on the surface of the $\mathrm{Si}$ target $\mathrm{I} \approx \approx 42 \mathrm{nA}(15.07 .2003)$

I - residual vacuum $\mathrm{P} \approx 1 * 10^{-5} \mathrm{~Pa}$, the surface is cleaned with an ion beam after $\mathrm{H}_{2} \mathrm{O}$ is injected into the chamber.

II - inlet into the $\mathrm{H} 2 \mathrm{O}$ chamber up to $\mathrm{P} \approx 2 * 10^{-3} \mathrm{~Pa}$. 
Fig.4.

The values of the dissociation energies for the $\mathrm{Sin}_{n} \mathrm{O}$ ${ }_{2 n+1}$ cluster with $n=(2-7)$, obtained by us according to the above-described method from the experiment, are in the range - 2.8-5.7 eV. Based on these data, the calculated values of the excitation energies were obtained, which lie in the range 3.68$17.58 \mathrm{eV}$ for clusters with $\mathrm{n}=(2-7)$ and $0.26-0.35 \mathrm{eV}$, respectively, per one oscillator. We also obtained mass spectra of negative cluster ions of silicon oxides $\mathrm{Me}_{n} \mathrm{O}_{-2 \mathrm{n}+1}$ and compared them with similar spectra, but with the addition of hydrogen - $\mathrm{Me}_{\mathrm{n}} \mathrm{O}$ ${ }_{2 n}+{ }_{1} \mathrm{H}_{k}(n=1-5, k=1-3)$ when in water. Comparisons of ion yields illustrated that 1 ) the yields of the $\mathrm{Sin}_{n} \mathrm{O}$ ${ }_{2 n+1}$ magic cluster with $\mathrm{H}_{2} \mathrm{O}$ and $\mathrm{O}_{2}$ puffs are increased compared to the spectrum without puffing, 2) hydrated oxides of the type $\mathrm{Sin}_{2 n+1} \mathrm{H}$-, $\mathrm{Si}_{n} \mathrm{O}_{2 n}+{ }_{1} \mathrm{H}_{-2}, \mathrm{Sin}_{n} \mathrm{O}_{2 \mathrm{n}+1} \mathrm{H}_{3}$, and the latter begins to appear only in magic clusters with $\mathrm{n} \geq 2$. In magic clusters, the peaks of one hydrated (hydride) oxide are somewhat higher in height than the previous peak of $\mathrm{Si}_{n} \mathrm{O}-2 \mathrm{n}+1$ oxide. In non-magic clusters $(\mathrm{n}=1)$, the peaks of oxides exceed those of hydrated oxides. The mass spectra were used to study the channels of fragmentation of the above-mentioned clusters in all possible stoichiometric directions. Hydrogenation is a simple method for stabilizing the silicon surface against oxidation and thus is important in microelectronics. Reaching an inactive silicon surface, ideally ending in hydrogen (hydrogen), has been accomplished in the last 40 years. However, oxidation still takes place even on hydrogen terminated atomistic flat silicon surfaces. Electrochemical hydrogen passivation for easily emitting silicon pores is an essential but unstable process. Recently silicon nanowires have been produced for large scale synthesis. An essential requirement for their widespread use has been fulfilled. This is both technological and scientific importance of finding ways to stabilize them, so as to skillfully avoid the problems of degradation and low photoluminescence. To achieve this goal, the study of hydrogenated silicon clusters in relation to their certain local structural stability should be urgent, but so far, such information is not attainable. In this experiment, we studied the reaction of an aqueous molecule on different hydrogenated silicon clusters in order to relate stability to the local hydrogen configuration, and to shed light on the way to achieve stability, nonreactivity of hydrogenated silicon structures. Since size effects often appear for properties such as energy gap when the size of cluster structures reaches nanometers, we focus our study on the effect of size on oxidation and thus on stability. In this work [2], models of hydrogenated surfaces $\mathrm{Si}$ (001) and $\mathrm{Si}(111)$ - $\mathrm{SigH} 14-\mathrm{SiH} 2$ and $\mathrm{Si}_{10 H} 15-\mathrm{SiH}_{3}$ were obtained, as well as smaller $\mathrm{Si}_{2} \mathrm{H}_{6}-\mathrm{SiH}_{2}$ and $\mathrm{Si}_{5} \mathrm{H}_{10}-\mathrm{SiH}_{2}, \mathrm{SiH}_{3}-\mathrm{SiH}_{3}$ and $\mathrm{Si}_{4} \mathrm{H}_{9}-\mathrm{SiH}_{3}$. These reactions were studied to develop a dimensional dependence of reactivity with water and the stability of local structures. The reaction starts from both sides and then a transition state (TS) is formed. The reaction is eventually completed by an $\mathrm{H}_{2}$ molecule that is released and forms $\mathrm{OH}$ attached to the silicon cluster. It turns out that the reaction can go through:

$$
\mathrm{Si}_{9} \mathrm{H}_{14}-\mathrm{SiH}_{2}+\mathrm{H}_{2} \mathrm{O} \rightarrow \mathrm{IC} \rightarrow \mathrm{TS} \rightarrow \mathrm{Si}_{9} \mathrm{H}_{14}-\mathrm{SiHOH}+\mathrm{H}_{2}
$$

The appearance of a weak intermediate complex of a similar reaction - IC, which is $1.4 \mathrm{kcal} / \mathrm{mol}$ lower than the reaction. In this case, the $\mathrm{O}-\mathrm{Si}_{1}$ distance = $4.054 \AA$. Thus, it is similar to the fact that the forces for this weak chelator are the dipole-dipole interaction. The system then has to go through a transient state TS in order to reach the product. In the case of TS with the O-Si $i_{1}$ bond, the distance between the attached $\mathrm{O}$ of the $\mathrm{H}_{2} \mathrm{O}$ atom and the attached silicone atom on the surface of $1.855 \AA$ is slightly longer than in the product; accordingly, the formed $\mathrm{H}_{1}-\mathrm{H}_{2}$ bond is much longer than the regular value in the product. Meanwhile, one of the two hydrogen atoms in water, $\mathrm{H}_{1}$, moves away from the oxygen atom, resulting in an $\mathrm{H}_{1}$ - $\mathrm{O}$ distance of 1.110 $\AA$, which is drawn longer than IC. The hydrogen atom $\mathrm{H}_{2}$ is $1.842 \AA$ away from the silicon atom. The energy barrier to this reaction is $44.5 \mathrm{kcal} / \mathrm{mol}$ in relation to the reactants. From the point of view of thermodynamics, this reaction is beneficial, because it is exothermic at $14.9 \mathrm{kcal} / \mathrm{mol}$. For other complexes - similar reactions. It is noticed that each of these reactions includes an intermediate complex with an energy of almost $2.3 \mathrm{kcal} / \mathrm{mol}$. With an expansion in the bunch size of the response of each sort of silicon-hydrogen arrangement, the energy hindrance diminishes (47-44), (44-42), everything shows an increment in the reactivity of the framework. Responses with $\mathrm{SiH}_{3}$ show a more modest energy obstruction than with $\mathrm{SiH}_{2}$. To give more positive help for the reactivity pattern, rate 
constants were determined at 1 atm. for a watery response inside different hydrogenated setups. For reactions on a dihydride, the calculated reaction rate for medium clusters shows a much larger increase in relation to small clusters, but can only increase slightly with increasing cluster size. Confirmation of the above may also appear from the analysis of their boundary orbitals. It has previously been found that the overlap between the highest occupied molecular orbitals (HOMO) of a molecule and the lowest unoccupied molecular orbitals (LUMO) can be determined by the nature of the chemical reaction. A smaller energy difference between HOMO of one molecule (electron donor) and LUMO of another (electron acceptor) might show a more preferable reaction. Thus, the tendency is the close ratio of HOMO and LUMO of individual hydrogen-silicon clusters, for which, with an increase in the cluster size, their HOMO generally moves forward and LUMO falls, as a result of which the energy gap (gap) decreases. Since the energy barrier size effect is well known for silicon clusters when dimensional changes are in the nanometer range, the correlation of reactivity with the well documented energy barrier size effect could provide an important implication for the size dependent reactivity found here. Based on small silicon clusters, it is expected that the reactivity and rate constants for large clusters will also stabilize for a given temperature and pressure.

\section{CONCLUSION}

The size-dependent reactivity found here should provide the most important scientific basis for the realization of non-reactive stable siliceous structures. We assume that such non-reactive stable structures can be achieved by the formation of uniform nanoscale structures (should be $<10 \mathrm{~nm}$, at which the specified size effect becomes significant). If this is the case, the oxidation found for porous silicon is the result of poor homogeneity and the small size of their nano structures is negligible. We hope that oxidation resulting from the same reactive local structures can lead to propagation of oxidation through oxidation of the backbone. Fortunately, one silicone structure is well hydrogenated and the surface reconstruction is removed, the reaction that takes place on the spine of the silicon structure becomes less possible, according to our study. Thus, in order to obtain a stable, non-reactive hydrogenated silicon structure, it is important to achieve non-rearranged structures uniformly coated with silicone dihydride, in particular, with a structural size of nanometers. Hydrogenated silicon structures show sizedependent reactivity and oxidation stability. With nano-sized hydrogenated silicon structure, in particular with dihydride finished nano-sized silicon structure, non-reactive stable nano-circuits are produced. Thus, the set of decay channels for $\mathrm{Sin}_{2} \mathrm{O}_{2 n}$ $+{ }_{1} \mathrm{H}_{\mathrm{k}}-(\mathrm{k}=1-3)$ clusters indicates their formation in parallel chains of combinatorial synthesis reactions in accordance with the mechanism $[9,10]$.

\section{REFERENCES}

1. N. Imanishi, S. Kyoh, A. Shimizu, M. Imai, A. Itoh. NIM B 135, 1998, 424.

2. I.A. Wojciechowski, U. Kutliev, Sh. Sun, Ch. Szakal, N. Winograd, B. J. Garrison. Appl. Surf. Scienc. 231-232 (2004) 72-77.

3. B. Hagenhoff, in J. S. Vickerman, D. Briggs (Eds.), ToF-SIMS: Surf. Analys. By Mass Spectr., IM Publ. And Surf. Spectra Lim., Manchester and Chichester, 2001, p. 285-308.

4. Chemical encyclopedia. M.: Soviet encyclopedia. 1988. T. 1.p. 285.

5. P.K.Khabibullaev, E.M. Bekmuratova, S.L. Pozharov. Electrostatic model of cluster ions and thermodynamic parameters of cluster formation reactions. Tashkent, FAN, 1984.142 p.

6. V. N. Kondrat'ev, E. E. Nikitin, A. I. Reznikov, S. Ya. Umansky. Thermal bimolecular reactions in gases. Moscow: Nauka, 1976.192 p.

7. P.P. Kulik, G.E. Norman, L.S.Polak. High energy chemistry. 10, No. 3 (1976) 203-220.

8. R.Q. Zhang, W.C. Lu, S.T. Lee. Appl. Phys. Lett. 80, No. 22 (2002) 4223.

9. N.Kh. Dzhemilev. // Surface.X-ray, synchrotron and neutron research. 2012. No. 8. p.28-34.

10. Dzhemilev N.Kh., Kovalenko S.F., Maksimov S.E., Tukfatullin O.F., Khozhiev Sh.T. // Surface. $\mathrm{X}$-ray., Synchrotron. and a neutron. issled. 2015. No. 4. P. 89. 